\title{
Formação e dinâmica da interface líquido-vapor simulada pelo método Lattice-Boltzmann
}

(Formation and dynamics of the liquid-vapor interface simulated by the Lattice-Boltzmann method)

\author{
Fabiano G. Wolf ${ }^{1}$, Luis O.E. dos Santos e Paulo C. Philippi \\ Laboratório de Meios Porosos e Propriedades Termofísicas, Departamento de Engenharia Mecânica, \\ Universidade Federal de Santa Catarina, Trindade, Florianópolis, SC, Brasil \\ Recebido em 24/8/2005; Aceito em 18/2/2006
}

\begin{abstract}
O método Lattice-Boltzmann (LB) tem sido utilizado como um modelo alternativo para a simulação computacional da dinâmica de fluidos regida pelas equações de Navier-Stokes. É fundamentado na equação de transporte de Boltzmann, que serve como base da teoria cinética. Devido ao seu grande potencial para a análise de problemas com geometrias complexas, tem sido amplamente aplicado para a descrição de escoamentos de fluidos com um ou mais componentes em meios porosos, principalmente para o estudo de fenômenos interfaciais. Neste trabalho, um modelo LB com potencial de interação entre partículas foi utilizado para a modelagem da interface líquido-vapor. Este método possibilita a simulação de equilíbrio de fases através de uma equação de estado que possui o comportamento semelhante à equação de van der Waals. O método também permite a inclusão de termos de interação entre partículas distintas, de modo que as interfaces líquido-sólido e vapor-sólido podem ser facilmente simuladas. Alguns resultados de simulação são apresentados para problemas que envolvem a transição de fase líquido-vapor, a coexistência de uma gota líquida com seu vapor e a molhabilidade de superfícies sólidas. Adicionalmente, o método é aplicado para o problema dinâmico de uma torneira gotejante. Os resultados obtidos mostram que o método pode ser utilizado para simulação de uma ampla variedade de sistemas complexos com relação às interfaces fluido-fluido e fluido-sólido.
\end{abstract}

Palavras-chave: interface líquido-vapor, Lattice-Boltzmann, transição de fase, molhabilidade, torneira gotejante.

The Lattice-Boltzmann (LB) method has been used as an alternative model for the computational simulation of fluid dynamics governed by Navier-Stokes equations. It is based on the Boltzmann transport equation, which works as the foundation of kinetic theory. Considering its suitability for complex geometry problems, it has been widely applied for the description of fluid flow with one or more components inside porous media, especially for interfacial phenomena studies. In this work, a LB model with an interaction potential among particles was used for modelling of the liquid-vapor interface. This method makes possible the simulation of phase equilibrium based on an equation of state similar to the van der Waals equation. The method allows the inclusion of interaction terms among different particles, so that liquid-solid and vapor-solid interfaces can be simulated. Some simulation results are presented to the problems involving the liquid-vapor phase transition, coexistence of a liquid droplet with its vapor and wettability on solid surfaces. Additionally, the method is applied to the dynamical problem of a dripping faucet. The obtained results show that the method can be used to simulate a large diversity of physical phenomena with respect to fluid-fluid and fluid-solid interfaces.

Keywords: liquid-vapor interface, Lattice-Boltzmann, phase transition, wettability, dripping faucet.

\section{Introdução}

Atualmente, o estudo de fenômenos interfaciais é assunto de grande interesse científico e econômico. Isso se deve ao constante desenvolvimento de métodos de simulação computacional e da capacidade crescente do poder de processamento. Desse modo, torna-se viável a possibilidade de simular diretamente a dinâmica molecular, regidas pelas leis do movimento de Newton $[1,2,3,4,5]$, e obter a dinâmica de interfaces, como

\footnotetext{
${ }^{1}$ E-mail: fgwolf@lmpt.ufsc.br.

Copyright by the Sociedade Brasileira de Física. Printed in Brazil.
}

por exemplo, líquido-vapor e vapor-sólido, vista como o resultado estatístico do comportamento coletivo molecular. Porém, este procedimento ainda é restrito a escalas de espaço e tempo diminutas, distantes daquelas encontradas em aplicações práticas. Da mesma forma, a utilização de métodos computacionais convencionais baseados em equações puramente macroscópicas também oferece desvantagens, pois são insensíveis à dinâmica microscópica envolvida, de modo que quantida- 
des físicas, como o ângulo de contato - ângulo formado entre a superfície do líquido e a superfície do sólido -, que poderiam ser modificadas por efeitos dinâmicos e/ou por outras características decorrentes da interação do fluido com o sólido, são mantidas constantes durante todo o experimento numérico.

$\mathrm{Na}$ tentativa de levar em consideração os aspectos essenciais da física pertinente em sistemas que envolvem diferentes tipos de interfaces de interação e evitar a grande quantidade de informação presente em métodos baseados na dinâmica molecular, modelos mesoscópicos de sistemas de partículas têm sido desenvolvidos.

O método Lattice-Boltzmann [6, 7, 8, 9] é baseado na discretização da equação mesoscópica de Boltzmann, que serve como base da teoria cinética dos gases, com a aproximação BGK [10] para o operador de colisão. Neste método, as distribuições de partículas são restritas a uma rede discreta, na qual cada sítio possui um número finito de velocidades discretas apontando para as sítios vizinhos, de modo que, além do espaço físico, o espaço de velocidades é discretizado. Apesar destas simplificações, este modelo discreto recupera adequadamente as equações de Navier-Stokes para dinâmica de fluidos em regime incompressível. Este fato evidencia que o comportamento macroscópico de fluidos independe da dinâmica microscópica complexa envolvida em tais sistemas [11].

Neste trabalho, o modelo mono-componente proposto por Shan e Chen [12, 13] é utilizado para a modelagem da interface líquido-vapor. Este modelo inclui um potencial de interação entre partículas, o que possibilita a simulação da coexistência de fases em equilíbrio, através de uma equação de estado que possui o comportamento semelhante à equação de van der Waals. Para um melhor acompanhamento da evolução dinâmica do sistema nas simulações, foram considerados apenas situações físicas bi-dimensionais para problemas que envolvem a transição de fase líquido-vapor, a coexistência de uma gota líquida com seu vapor e a molhabilidade de superfícies sólidas. Adicionalmente, o método é aplicado para o problema dinâmico de uma torneira gotejante, no qual a influência das condições iniciais e de contorno pôde ser observada.

\section{Método Lattice-Boltzmann}

O método Lattice-Boltzmann (LB) [6, 7, 8, 9] é um método mesoscópico para a descrição de um sistema mecânico de partículas. Embora, historicamente, tenha sido originado de autômatos celulares, como o LatticeGas Automata [14, 11], recentemente foi mostrado [15] que o método LB pode ser considerado como uma forma discreta especial da equação de Boltzmann para gases diluídos. Como a equação de Boltzmann, os modelos LB são formulados na escala mesoscópica, na qual a descrição do sistema não é feita diretamente a partir das partículas individuais - como é comum em méto- dos baseados em dinâmica molecular $[1,2,3,4,5]$ - mas através da função distribuição de partículas, $f_{i}(\mathbf{x}, t)$, que representa o valor esperado do número de partículas com velocidade $\mathbf{c}_{\mathbf{i}}$ no sítio $\mathbf{x}$ e no tempo $t$, onde $i=0 \ldots b$. Nos modelos LB, as partículas são restritas a uma rede discreta, de forma que cada grupo de partículas pode se deslocar somente num número finito $b$ de direções e com um número limitado de velocidades (ver Fig. 1). Assim, tanto o espaço físico quanto o espaço de velocidades são discretizados. As propriedades macroscópicas locais como massa total (a massa $m$ de cada partícula é assumida unitária $), \rho(\mathbf{x})$, e quantidade de movimento, $\rho(\mathbf{x}) \mathbf{u}(\mathbf{x})$, podem ser obtidas da função distribuição da seguinte forma:

$$
\begin{gathered}
\rho(\mathbf{x})=\sum_{i} f_{i} \\
\rho(\mathbf{x}) \mathbf{u}(\mathbf{x})=\sum_{i} f_{i} \mathbf{c}_{i} .
\end{gathered}
$$

Devido ao efeito das colisões, a função distribuição de partículas $f_{i}(\mathbf{x}, t)$ é modificada em cada sítio da rede durante o intervalo de tempo $\Delta t$. A equação de evolução de que descreve esta mudança é dada por:

$$
f_{i}\left(\mathbf{x}+\mathbf{c}_{i} \Delta t, t+\Delta t\right)-f_{i}(\mathbf{x}, t)=\Omega_{i},
$$

onde $\Omega_{i}$ é o operador de colisão. Este deve ser escolhido de tal modo a conservar a massa e a quantidade de movimento (além da energia total em problemas nãoisotérmicos), então:

$$
\begin{gathered}
\sum_{i} \Omega_{i}=0 \\
\sum_{i} \Omega_{i} \mathbf{c}_{i}=0 .
\end{gathered}
$$

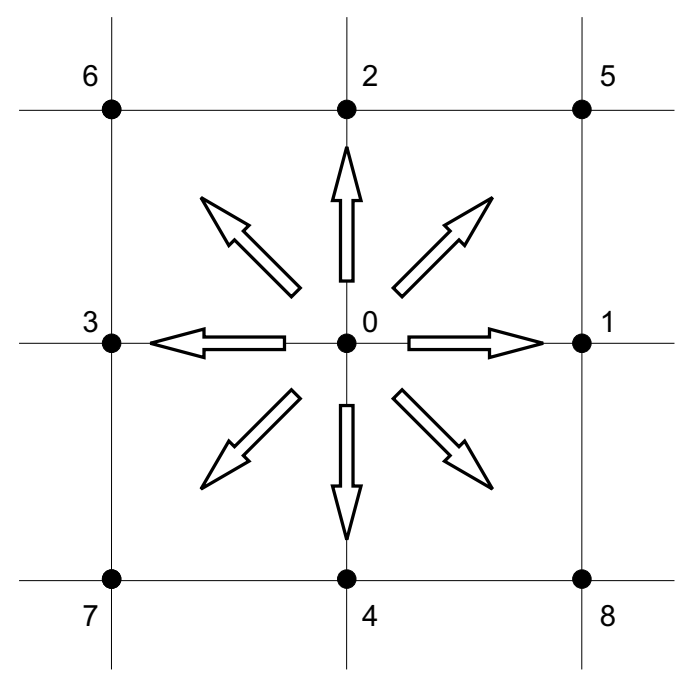

Figura 1 - Rede D2Q9 (duas dimensões e nove velocidades). Note que as partículas podem possuir nove velocidades, com as seguintes magnitudes: $\left|\mathbf{c}_{0}\right|=0,\left|\mathbf{c}_{1,2,3,4}\right|=1 \mathrm{e}\left|\mathbf{c}_{5,6,7,8}\right|=\sqrt{2}$. 
A forma mais simples de considerar o efeito das colisões entre partículas é utilizar o operador introduzido por Bhatnagar, Gross e Krook (BGK) [10]. Este descreve a colisão como um processo de relaxação para o estado de equilíbrio local e é dado por:

$$
\Omega_{i}=-\frac{\Delta t}{\tau}\left(f_{i}-f_{i}^{(e q)}\right),
$$

onde $\tau$ é o tempo de relaxação e $f_{i}^{(e q)}$ é a distribuição de equilíbrio local de partículas. Logo, a equação mesoscópica governante para o método LB será:

$$
f_{i}\left(\mathbf{x}+\mathbf{c}_{i} \Delta t, t+\Delta t\right)-f_{i}(\mathbf{x}, t)=-\frac{\Delta t}{\tau}\left(f_{i}-f_{i}^{(e q)}\right) .
$$

Após a colisão entre partículas, a equação de evolução (5) exige que a informação local em $\mathbf{x}$ e em $t$, $f_{i}(\mathbf{x}, t)$, seja transmitida para o sítio vizinho $\mathbf{x}+\mathbf{c}_{i} \Delta t$ no tempo $t+\Delta t$, o que é feito pela etapa de propagação.

O comportamento macroscópico da Eq. (5) pode ser determinado através da escolha adequada da distribuição de equilíbrio, $f_{i}^{(e q)}=f_{i}^{(e q)}(\rho, \mathbf{u})$. No presente caso, busca-se resgatar a dinâmica macroscópica de fluidos regida pelas equações de Navier-Stokes. Para esse fim, a distribuição de equilíbrio é escolhida como [6],

$$
\begin{gathered}
f_{i}^{(e q)}(\mathbf{x})=\rho(\mathbf{x})\left[\frac{1-d_{0}}{b}+\frac{D}{c^{2} b} \mathbf{c}_{i} \cdot \mathbf{u}+\right. \\
\left.\frac{D(D+2)}{2 c^{4} b} \mathbf{c}_{i} \mathbf{c}_{i}: \mathbf{u} \mathbf{u}-\frac{D}{2 b c^{2}} \mathbf{u}^{2}\right], \\
f_{0}^{(e q)}(\mathbf{x})=\rho(\mathbf{x})\left[d_{0}-\frac{\mathbf{u}^{2}}{c^{2}}\right],
\end{gathered}
$$

onde $d_{0}$ é a fração de partículas em repouso, $c=\left|\mathbf{c}_{i}\right| \mathrm{e}$ $D$ é a dimensão espacial.

Através do método multi-escala de ChapmanEnskog [16] pode ser mostrado que o sistema descrito acima recupera as seguintes equações governantes em regime incompressível:

$$
\begin{gathered}
\nabla \cdot \mathbf{u}=0 \\
\frac{\partial \mathbf{u}}{\partial t}+\mathbf{u} \cdot \nabla \mathbf{u}=-\frac{\nabla P}{\rho}+\nu \nabla^{\mathbf{2}} \mathbf{u},
\end{gathered}
$$

onde $P=\frac{c^{2}\left(1-d_{0}\right)}{D} \rho$ e $\nu=\frac{c^{2}}{D+2}(\tau-1 / 2)$ são a pressão termodinâmica e a viscosidade cinemática, respectivamente. As equações acima representam a equação da continuidade e a equação de Navier-Stokes, para a conservação da massa e da quantidade de movimento.

Próximo à superfícies sólidas, a condição de contorno bounce-back é imposta sobre aquelas partículas que entram em contato com os sítios sólidos. Esta condição de contorno consiste em inverter a quantidade de movimento das partículas exatamente para direção contrária de contato, como pode ser visto na Fig. 2. Embora a condição bounce-back seja simples, esta garante a condição de não-escorregamento, na qual a velocidade do fluido junto à parede é nula.

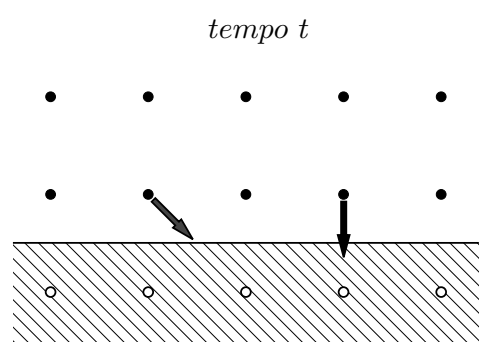

(a)

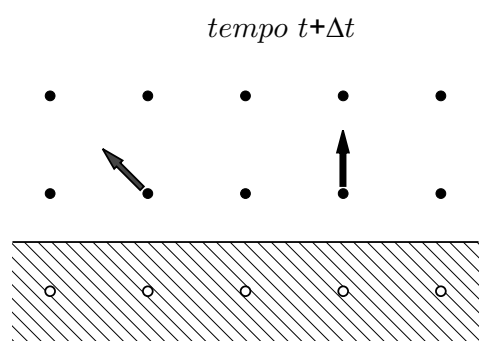

(b)

Figura 2 - Ilustração da condição de contorno bounce-back para as partículas que colidem com sítios sólidos.

\subsection{Modelo com potencial de interação}

Buscando se aproximar da realidade física no que se refere às interações microscópicas entre as moléculas (ou átomos) de um fluido, Shan e Chen [12] introduziram no método LB um potencial de interação entre as partículas constituintes do fluido. Dessa forma, além da separação entre fluidos imiscíveis (que não se misturam naturalmente, como por exemplo, água e óleo), o modelo é capaz de reproduzir a transição de fase líquidovapor, tornando possível a simulação da coexistência de duas fases distintas em equilíbrio, no qual a densidade automaticamente identifica ambas as fases. Para esse fim, os autores introduziram a seguinte energia potencial de interação entre pares de partículas localizadas em $\mathbf{x}$ e $\mathbf{y}$ :

$$
V(\mathbf{x}, \mathbf{y})=\varphi(\mathbf{x}) G_{i}(\mathbf{x}, \mathbf{y}) \varphi(\mathbf{y})
$$

onde $G_{i}(\mathbf{x}, \mathbf{y})=\mathcal{G}$ para $|\mathbf{y}-\mathbf{x}|=\sqrt{2}, G_{i}(\mathbf{x}, \mathbf{y})=2 \mathcal{G}$ para $|\mathbf{y}-\mathbf{x}|=1$ e $G_{i}(\mathbf{x}, \mathbf{y})=0$ para dois sítios que não sejam primeiros vizinhos. A função $\varphi$ é dependente da densidade de partículas local. Note que a magnitude de $\mathcal{G}$ determina a intensidade do potencial de interação entre as partículas em $\mathbf{x}$ e $\mathbf{y}$, enquanto seu sinal define se o potencial é atrativo ou repulsivo.

A força resultante desse potencial é dada por:

$$
\mathbf{F}_{\sigma}(\mathbf{x})=-\varphi(\mathbf{x}) \sum_{i} G_{i} \varphi\left(\mathbf{x}+\mathbf{c}_{\mathbf{i}} \Delta t\right) \mathbf{c}_{\mathbf{i}} .
$$

Um forma semelhante de potencial de interação foi utilizada por Martys e Chen [17] para o caso de um fluido 
interagindo com um sólido. Neste caso, a força resultante no sítio $\mathbf{x}$ no tempo $t$ é dada por:

$$
\mathbf{F}_{s}(\mathbf{x})=-\rho(\mathbf{x}) \sum_{i} W_{i} s\left(\mathbf{x}+\mathbf{c}_{\mathbf{i}} \Delta t\right) \mathbf{c}_{\mathbf{i}},
$$

onde $W_{i}(\mathbf{x}, \mathbf{y})=\omega$ para $|\mathbf{y}-\mathbf{x}|=\sqrt{2}, W_{i}(\mathbf{x}, \mathbf{y})=2 \omega$ para $|\mathbf{y}-\mathbf{x}|=1$ e $W_{i}(\mathbf{x}, \mathbf{y})=0$ para dois sítios que não sejam primeiros vizinhos. Na expressão acima, $s=1$ representa que o sítio vizinho é sólido, enquanto que $s=0$ representa que sítio vizinho é fluido.

Desse modo, a velocidade local, $\mathbf{u}(\mathbf{x})$, na distribuição de equilíbrio 6 é modificada devido à ação das forças existentes da seguinte forma:

$$
\mathbf{u}^{\prime}=\mathbf{u}+\frac{\tau}{\rho}\left(\mathbf{F}_{\sigma}+\mathbf{F}_{s}+\mathbf{F}_{g}\right) .
$$

onde $\mathbf{F}_{g}=\rho g \mathbf{j}$ é a força gravitacional.

Novamente, o método de Chapman-Enskog permite determinar o comportamento macroscópico deste sistema. A nova equação governante descreve um fluido não-ideal, cuja equação de estado (ver Fig. 3) é dada por:

$$
P=\frac{c^{2}}{D}\left[\left(1-d_{0}\right) \rho+\frac{\mathcal{G} b}{2} \varphi^{2}(\rho)\right]
$$

Segundo Shan e Chen [12], para um processo isotérmico termodinamicamente consistente, $\varphi(\rho)$ deve ser escolhido como:

$$
\varphi(\rho)=\varphi_{0} \exp \left(-\rho_{0} / \rho\right),
$$

onde $\rho_{0}$ e $\varphi_{0}$ são constantes arbitrárias. Observando a equação de estado acima, nota-se que o parâmetro $-\left(1-d_{0}\right) / \mathcal{G}$ tem o mesmo papel que a temperatura na teoria de van der Waals, uma vez que a temperatura pode ser modificada tanto pela fração de partículas em repouso quanto pela intensidade do campo de interação [12].

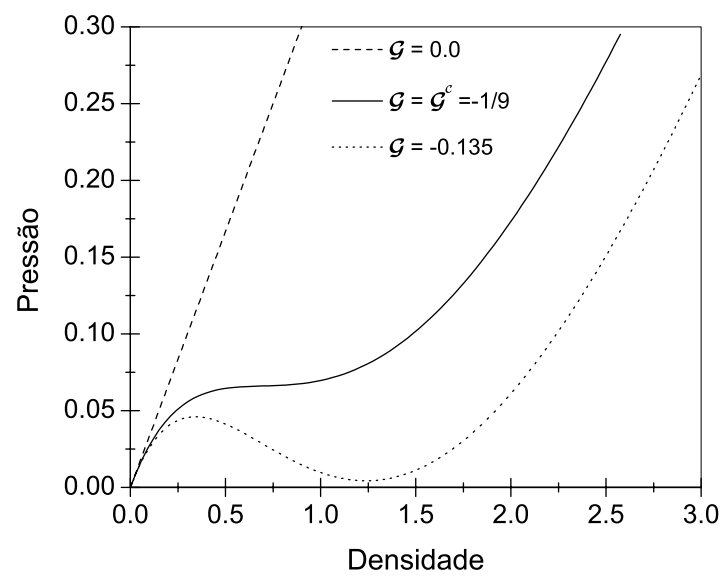

Figura 3 - Pressão vs. densidade para diferentes valores de $\mathcal{G}$. O valor crítico $\mathcal{G}_{c}$ pode ser obtido a partir de $\partial P / \partial \rho=\partial^{2} P / \partial \rho^{2}=0$ no ponto crítico.

\section{Resultados e discussão}

Para testar a capacidade do modelo de Shan e Chen [12] para a simulação de diferentes interfaces, foram abordados tópicos que envolvem tanto a modelagem da interface líquido-vapor quanto das interfaces líquido-sólido e vapor-sólido. A menos que seja indicado, as simulações foram conduzidas numa rede D2Q9 com os parâmetros $\tau=1,0$ e $\mathcal{G}=-0,15$, resultando na razão de densidades de $\rho_{L} / \rho_{V} \simeq 20$. As unidades utilizadas estão em unidades de rede, na qual a densidade de massa, a distância e o tempo são descritas em termos de partículas por sítio $(m=1)$, espaços de rede $(\Delta x=1)$ e passos de tempo $(\Delta t=1)$, respectivamente. Seguindo [12], utiliza-se a função $\varphi(\rho)=1-\exp (-\rho)$ no lugar da Eq. (14). Essas funções apresentam comportamento similar, porém esta escolha leva à melhoria da estabilidade nas simulações. Nas fronteiras do domínio de simulação foram impostas condições de contorno pediódicas em todas as direções.

\subsection{Tensão superficial}

A tensão superficial de líquidos resulta do desbalanço de forças intermoleculares em regiões interfaciais, nas quais existe a variação brusca da densidade. Desse modo, a força resultante numa molécula próxima à interface líquido-vapor é diferente daquela sobre uma molécula que se encontra em uma região completamente homogênea (na qual a força resultante é nula). Para uma gota em equilíbrio com seu vapor, o efeito da tensão superficial é aumentar a pressão interna da gota, de acordo com a lei de Young-Laplace:

$$
P_{i}-P_{e}=(D-1) \frac{\sigma}{R}
$$

onde $P_{i}, P_{e}, \sigma, R$ e $D$ representam a pressão interna e externa, a tensão superficial, o raio da gota e a dimensão espacial, respectivamente.

A lei de Young-Laplace diz que a pressão interna de uma gota pode ser modificada tanto pela variação da tensão superficial quanto pela variação do raio da gota. Em princípio, para um processo isotérmico, a tensão superficial não varia. Então, para testar a validade da lei de Young-Laplace para o modelo exposto acima, podese variar o raio da gota e medir a variação de pressão correspondente. A partir do gráfico $\left(P_{i}-P_{e}\right) \times 1 / R$, pode-se determinar a tensão superficial através do coeficiente angular da reta. Os resultados obtidos com o método LB são mostrados na Fig. 4. Nota-se que a linearidade entre a diferença de pressão e o inverso do raio é observada, resultando na tensão superficial $\sigma \simeq 0,086$ 


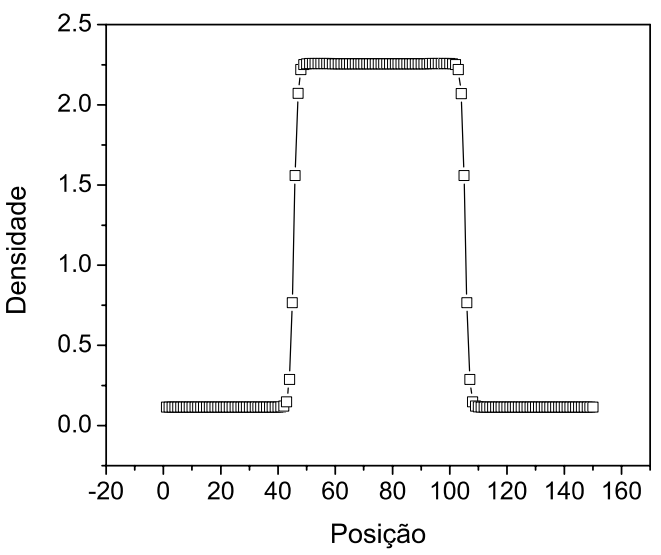

(a)

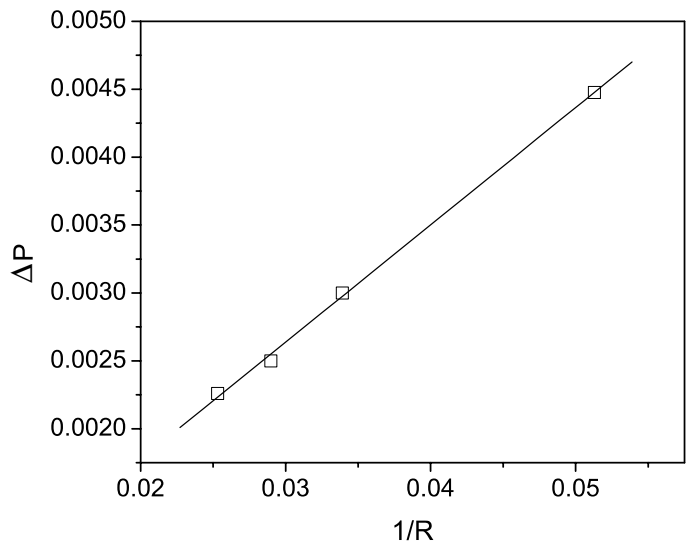

(b)

Figura 4 - Em (a) é mostrado o perfil de densidades de uma gota imersa em seu vapor para uma rede com tamanho de $150 \times 150$ sítios. (b) Verificação da lei de Young-Laplace para o modelo descrito no texto.

\subsection{Transição de fase}

A transição de fase líquido-vapor refere-se ao processo pelo qual líquido pode ser transformado em vapor (ou vice-versa) por meio da modificação de variáveis do sistema, como pressão, temperatura e volume. Por exemplo, é possível transformar o vapor aquecido contido num êmbolo em líquido por meio da diminuição do volume num processo a temperatura constante. Da mesma forma, o vapor aquecido pode ser transformado em líquido pela diminuição isovolumétrica da temperatura até um determinado valor crítico, no qual pequenas gotas líquidas começam a aparecer no recipiente. Fisicamente, a diminuição da temperatura faz com que a energia cinética das moléculas e o efeito das colisões diminua até um limite no qual as forças intermoleculares tornam-se dominantes e acabam por agrupar as moléculas em regiões específicas. Uma vez que a presença de forças intermoleculares pode levar à transição de fase, é presumível que o modelo LB descrito acima também possa exibir este tipo de comportamento para determinados valores críticos. Como citado anteriormente, no modelo de Shan e Chen, o parâmetro que tem o mesmo papel da temperatura é dado por $-\left(1-d_{0}\right) / \mathcal{G}$. Desse modo, a temperatura pode ser modificada tanto pela fração de partículas em repouso, $d_{0}$, quanto pela intensidade do potencial de interação dada por $\mathcal{G}$. Por razões de estabilidade numérica, freqüentemente assume-se $d_{0}=1 / 3$. Logo, somente a modificação de $\mathcal{G}$ permitirá simular o efeito da temperatura no sistema.

Para fazer a simulação da transição de fase líquidovapor usando o modelo LB, uma rede de $200 \times 200$ sítios foi preenchida com a densidade local $\rho=\rho_{0}(1+z)$, onde $\rho_{0}$ é a densidade de referência e $z$ é uma perturbação aleatória pertencente ao intervalo $[-0,01,+0,01]$. Esta perturbação estatística é necessária, pois induzirá ao processo de transição de fase em condições favoráveis. $\mathrm{O}$ valor crítico $\mathcal{G}_{c}$, a partir do qual o sistema alcança as condições favoráveis para a transição de fase, pode ser obtido a partir da equação de estado, lembrando que este é um ponto de inflexão (ver Fig. 3):

$$
\left(\frac{\partial P}{\partial \rho}\right)_{\mathcal{G}_{c}, \rho_{c}}=\left(\frac{\partial^{2} P}{\partial \rho^{2}}\right)_{\mathcal{G}_{c}, \rho_{c}}=0 .
$$

Resolvendo as equações acima, obtêm-se:

$$
\mathcal{G}_{c}=-\frac{4\left(1-d_{0}\right)}{b}=-\frac{1}{9}
$$

$$
\rho_{c} \simeq 0,693 .
$$

Na determinação dos valores críticos acima, foi considerado que a rede D2Q9 é uma projeção no espaço bidimensional de uma rede hipercúbica de face centrada (FCHC) [18], na qual $D=4$ e $b=24$.

Inicialmente, verifica-se a partir da simulação, que a separação das fases é rápida, porém torna-se mais lenta devido à grande quantidade de gotículas competindo por aquelas partículas que ainda se encontram na fase gasosa. Na Fig. 5, pode ser visto que as gotas de raio menor são mais instáveis em relação as gotas maiores. Isso se deve aos efeitos da tensão superficial sobre a interface de maior curvatura, que leva a maior diferença de pressão em gotas menores, de modo que estas tendem a evaporar antecipadamente, enquanto que as gotas maiores tendem a crescer. Na Fig. 6 é mostrado o comportamento das densidades das fases líquida e vapor em função da temperatura definida para o modelo. Para obtenção desta curva de coexistência, uma interface plana foi inicializada perpendicularmente ao eixo $y$ para cada valor de $\mathcal{G}$, numa rede com $5 \times 600$ sítios. No equilíbrio, as densidades da fase líquida e vapor foram determinadas através do perfil de densidades. Como previsto teoricamente, o valor crítico de $\mathcal{G}_{c} \simeq-1 / 9$ é confirmado pelas simulações, visto a tendência observada. 


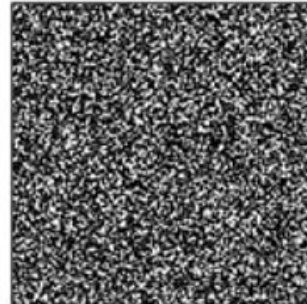

(a)

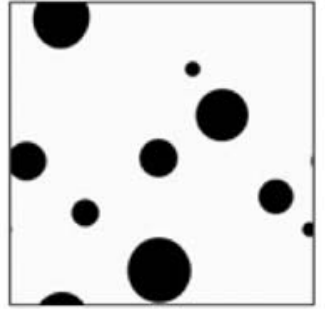

(e)

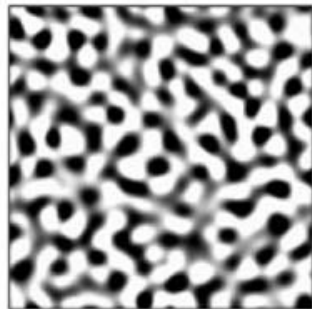

(b)

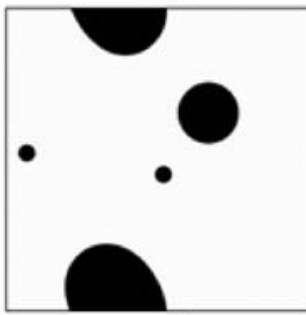

(f)

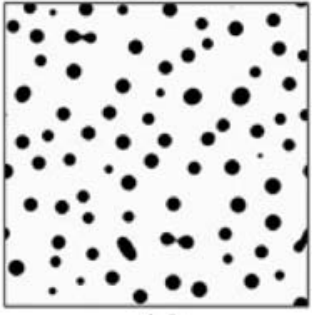

(c)

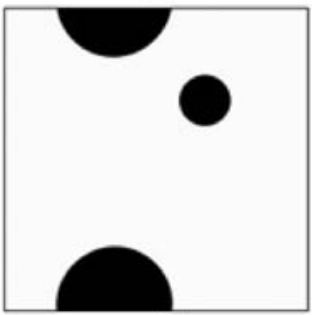

(g)

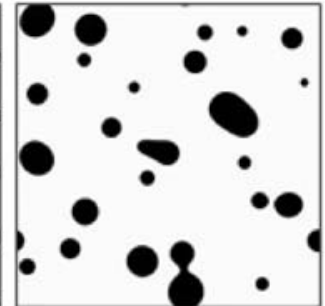

(d)

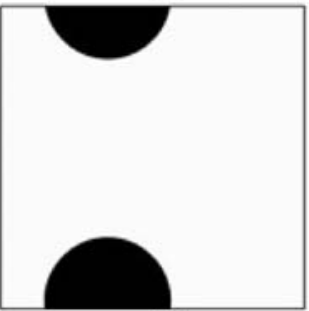

(h)

Figura 5 - Transição de fase simulada através do modelo LB para $\rho_{0}=1,2$ e $\mathcal{G}=-0,15$. A evolução do processo se dá de (a) a (h), no qual as regiões escuras apresentam a maior densidade, sendo identificadas como a fase líquida.

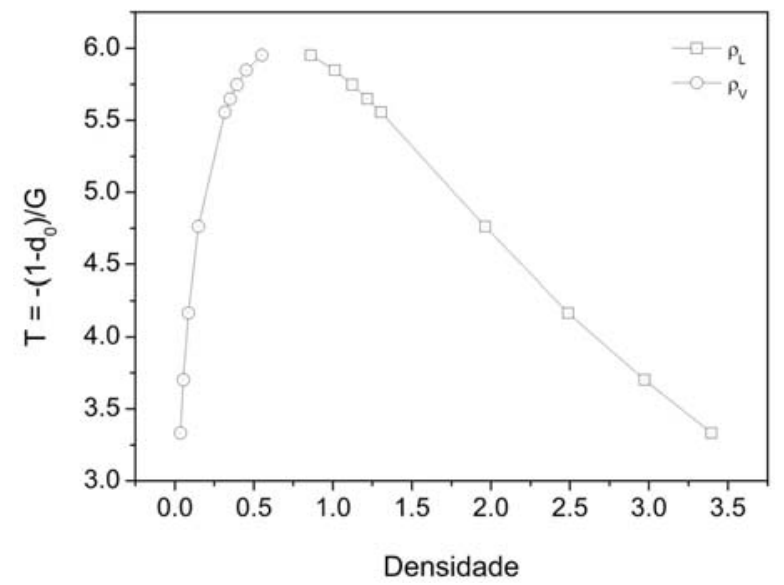

Figura 6 - Curva de coexistência de fases obtidas a partir de simulações com interface líquido-vapor plana. Verifica-se que a temperatura crítica prevista teoricamente, $-\left(1-d_{0}\right) / \mathcal{G}_{c}=6$, é confirmada pela tendência observada.

\subsection{Molhabilidade}

Num sentido mais amplo, a interação que ocorre entre dois fluidos e um sólido é chamada de molhabilidade. Essa propriedade é o resultado das interações moleculares existentes entre ambos os fluidos (por exemplo, líquido e vapor) e o substrato sólido. Quando líquido e vapor, estão em contato entre si, juntamente com a superfície sólida, observa-se a existência de uma linha comum para as três fases, conhecida como linha de contato. Essa configuração origina a definição do ângulo de contato $\theta$, como sendo o ângulo resultante entre a linha tangente à interface que separa o líquido e o vapor e a linha paralela à superfície do sólido, como mostrado na Fig. 7.

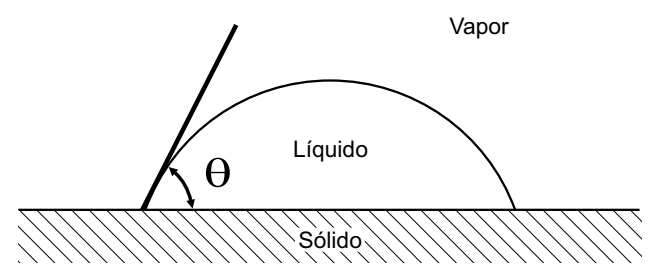

Figura 7 - Ilustração do ângulo de contato para uma gota molhante sobre uma superfície sólida.

O ângulo de contato representa um papel fundamental em fenômenos capilares, por exemplo, quando um tubo capilar fino é colocado em contato com uma superfície líquida. Neste caso, observa-se que se $\theta<90^{\circ}$ (típico do sistema ar-água-vidro), o líquido invadirá o capilar até que a força capilar seja equilibrada pelo peso da coluna líquida formada com a invasão, diz-se então que o líquido é molhante para aquele sólido. Porém, se $\theta>90^{\circ}$ (típico do sistema ar-mercúriovidro), observa-se que o líquido é repelido pelo capilar, originando uma depressão na superfície líquida, diz-se então que o líquido é não-molhante para aquele sólido. Quando $\theta=90^{\circ}$, as forças capilares são nulas e nada ocorre.

No modelo LB, a relação entre forças de coesão (interações fluido-fluido) e adesão (interações fluidosólido), que determina o ângulo de contato, pode ser simulada através da introdução das forças dadas pelas Eqs. (11) e (12), respectivamente. Para esse fim, uma gota líquida em contato com uma superfície sólida foi inicializada numa rede com $300 \times 78$ sítios para diferentes valores de $\omega$. Após alcançado o equilíbrio mecânico, 
o ângulo de contato foi medido a partir da seguinte relação (que assume aproximação esférica):

$$
\tan \left(\frac{\theta}{2}\right)=\frac{2 H}{D}
$$

onde $H$ e $D$ são a altura e o diâmetro da base da gota, respectivamente.

Os resultados obtidos são mostrados nas Figs. 8 e 9. A partir da variação de $\omega$, verifica-se que qualquer ângulo de contato entre 0 e $\pi$ pode ser simulado. Notase que o aumento das forças de adesão leva a um menor ângulo de contato em concordância com métodos baseados em dinâmica molecular [1, 4]. Desse modo, uma ampla variedade de fenômenos capilares pode ser estudada a partir do modelo, especialmente em geometrias complexas que apresentam grande variabilidade espacial, nos quais o processo de invasão capilar pode ser extremamente complicado e difícil de ser simulado através de métodos convencionais baseados em equações puramente macroscópicas.

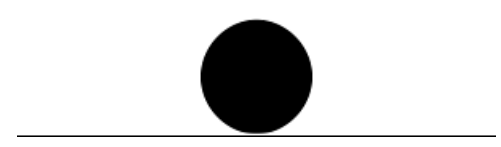

(a)

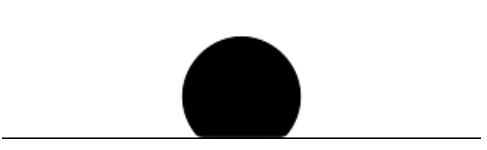

(b)

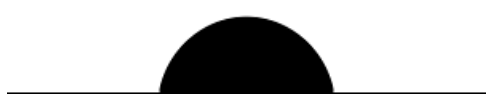

(c)

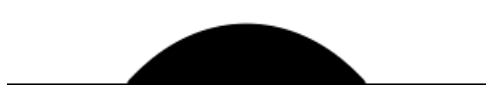

(d)

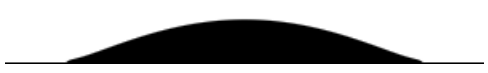

(e)

Figura 8 - Configurações de equilíbrio para uma gota em contato com uma superfície sólida para diferentes valores de $\omega$. De (a) até (e) os seguintes $\omega^{\prime} s$ foram ajustados: $-0,0200,-0,0350$, $-0,0500,-0,0575$ e $-0,0650$. Nota-se que o aumento das forças de adesão leva a um menor ângulo de contato.

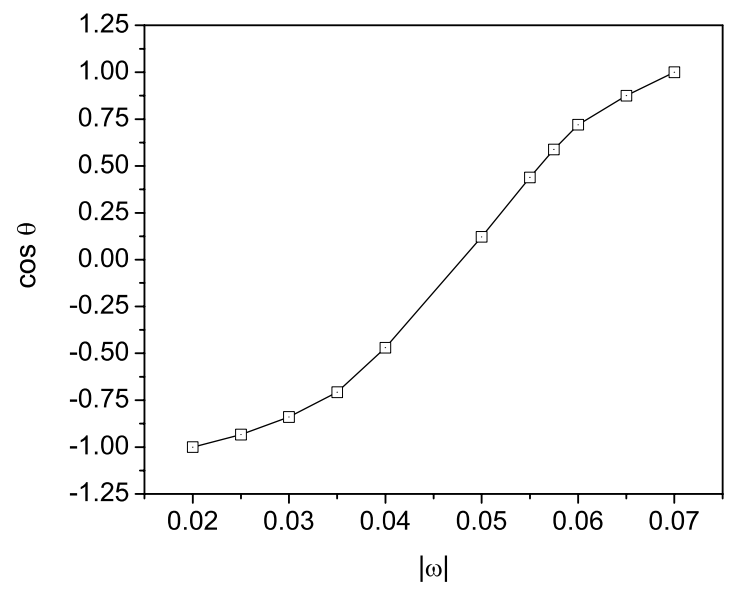

Figura 9 - Dependência do cosseno do ângulo de contato com a intensidade das forças de adesão.

\subsection{Um exemplo dinâmico: A torneira gote- jante}

A fim de demostrar a flexibilidade do método LB para a simulação de fenômenos dinâmicos, considera-se uma situação comumente encontrada no dia-a-dia: a torneira gotejante. A observação da dinâmica de gotejamento envolvida nesta aparentemente simples situação cotidiana, tem demostrado que a modelagem deste fenômeno pode ser complicada e apresentar comportamento caótico [19]. Nota-se que dependendo da vazão do líquido, que pode ser modificada pela abertura ou fechamento da torneira, o gotejamento pode variar da formação e ruptura regular de gotas, passando por um regime irregular até tornar-se um filamento contínuo de líquido.

Para simular a torneira gotejante, uma rede com $125 \times 255$ sítios foi inicializada com uma porção de líquido $\left(\rho_{L}=2,235\right)$ e outra de vapor $\left(\rho_{V}=0,112\right)$ localizadas na parte inferior e superior, respectivamente. Uma placa sólida foi colocada na base inferior, contendo um orifício com 15 espaços de rede de largura. O parâmetro que determina a intensidade das forças de adesão, $\omega$, foi ajustado para $-0,02$, para o qual $\theta=170^{\circ}$. Como são utilizadas condições periódicas de contorno em todas as direções, as partículas de líquido que saem pelo orifício são reinjetadas na parte superior, imitando assim, a torneira gotejante. Devido às forças capilares, o líquido deve ser forçado a fluir através do orifício, o que é feito utilizando a força gravitacional com $g=-8 \times 10^{-5}$ (unidades de rede). A sequência da formação e ruptura regular de gotas líquidas numa torneira é mostrada na Fig. 10. A partir da sequência de imagens obtida pela simulação, verifica-se que a formação de uma gota inicia-se lentamente através do orifício. A ruptura da primeira gota dá-se somente após o contato inicial da gota e a superfície líquida na parte inferior. Porém, as gotas que se formam posteriormente rompem-se depois de um tamanho crítico ser alcançado, 
no qual o peso da gota é suficientemente grande para diminuir a espessura do filamento contínuo que conecta a torneira à gota. Em seguida ao regime transitório inicial, a formação das gotas torna-se regular, seguindo aproximadamente a mesma dinâmica, pelo menos dentro do intervalo de tempo de simulação acompanhado.

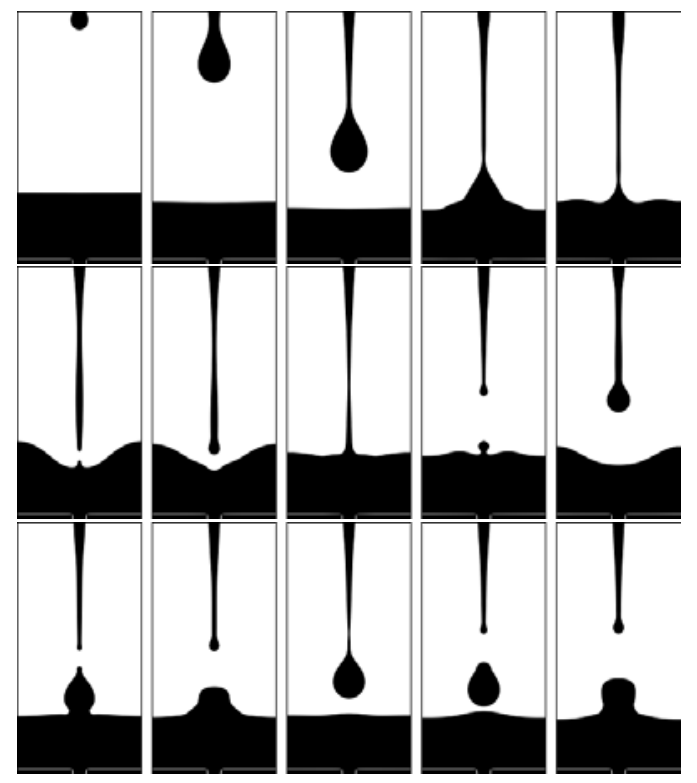

Figura 10 - Sequência da formação e ruptura regular de gotas líquidas numa torneira com orifício de 15 espaços de rede. As regiões escuras correspondem a fase líquida. O processo desenvolvese da esquerda para direita e de cima para baixo.

Com o intuito de mostrar a influência da condições iniciais e de contorno no gotejamento de uma torneira, a largura do orifício pelo qual a fase líquida flui foi modificado para 19 espaços de rede. Os resultados obtidos são mostrados na Fig. 11. Nota-se que tal alteração modificou completamente o regime do escoamento, fazendo com que aquela formação e ruptura regular de gotas fosse transformada na formação de um filamento de líquido contínuo entre a "torneira" e a superfície líquida.

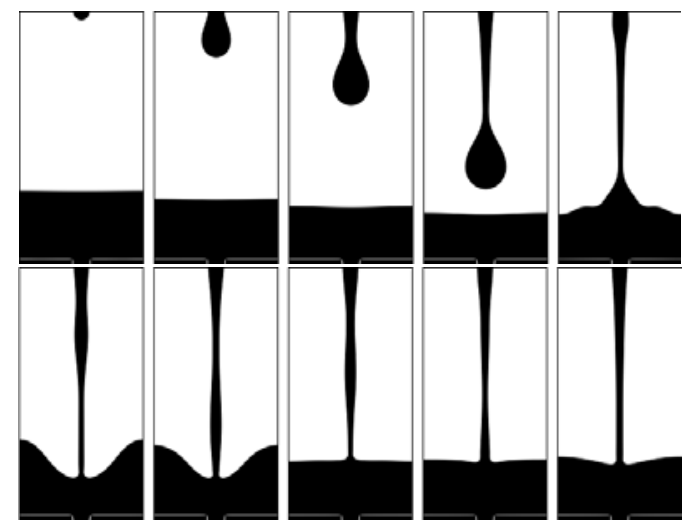

Figura 11 - Sequência da formação de um filamento de líquido numa torneira com orifício de 19 espaços de rede. As regiões es- curas correspondem a fase líquida. O processo desenvolve-se da esquerda para direita e de cima para baixo.

Pode-se observar a riqueza de formas superficiais que existem no processo de formação de gotas numa torneira, mostrando o motivo pelo qual tal problema tem sido visto como um fenômeno complexo. A simples modificação da "largura da torneira", o que altera a vazão de líquido, mostra como o regime é fortemente influenciado pelas condições iniciais e de contorno que antecedem a formação da primeira gota. Estas simulações mostram que o método LB pode prontamente servir como uma ferramenta auxiliar para o estudo de fenômenos físicos cujo entendimento completo ainda não está disponível.

\section{Conclusão}

Neste trabalho, mostra-se alguns exemplos da utilização do método Lattice-Boltzmann para a simulação de fenômenos físicos que envolvem as interfaces líquido-vapor, líquido-sólido e vapor-sólido. Embora apenas sistemas simples tenham sido considerados, fica evidente que o método é capaz de simular uma grande gama de fenômenos complexos. Essa flexibilidade é devida ao fato que propriedades físicas macroscópicas, como tensão superficial e ângulo de contato, serem obtidas como o resultado das interações mesoscópicas entre aglomerados de partículas. Porém, uma análise quantitativa aprofundada para problemas específicos é ainda necessária para realmente validar o modelo em questão. Mais importante ainda é a consideração de efeitos térmicos nas simulações mostradas. Como pode ser concluído através do texto, a temperatura no modelo considerado, não é bem definida, e acaba sendo apenas um parâmetro pré-definido, que não influencia localmente o sistema dinâmico; o que não é desejável, pois sabe-se que na transição líquido-vapor, há liberação de calor, uma vez que as moléculas na fase vapor apresentam maior mobilidade do que na fase líquida. Logo, a consideração coerente de efeitos térmicos é o fator determinante para se estabelecer a consistência física do método LatticeBoltzmann para a modelagem de fenômenos complexos, freqüentemente encontrados na natureza.

\section{Agradecimentos}

Ao suporte financeiro dado pela Agência Nacional do Petróleo (ANP), à Financiadora de Estudos e Projetos (FINEP) e ao Conselho Nacional de Desenvolvimento Científico e Tecnológico ( $\mathrm{CNPq})$.

\section{Referências}

[1] J.H. Sikkenk, J.O. Indekeu and J.M.J. van Leeuwen, Phys. Rev. Lett. 59, 98 (1987). 
[2] J. Koplik, J.R. Banavar and J.F. Willemsen, Physical Review Letters 60, 1282 (1988).

[3] P.A. Thompson and M.O. Robbins, Physical Review Letters 63, 766 (1989).

[4] M.J.P. Nijmeijer, C. Bruin and A.F. Bakker, Phys. Rev. A 42, 6052 (1990).

[5] S. Maruyama, T. Kimura and M. Lu, Thermal Science and Engineering 10, 23 (2002).

[6] Y.H. Qian, D. d'Humières and P. Lallemand, Europhysics Letters 17, 479 (1992).

[7] H. Chen, S. Chen and W.H. Matthaeus, Physical Review A 45, R5339 (1992).

[8] Sauro Succi, The Lattice Boltzmann Equation for Fluid Dynamics and Beyond, (Oxford University Press, Oxford, 2001), $288 \mathrm{p}$.

[9] D. Wolf-Gladrow, Lattice Gas Cellular Automata and Lattice Boltzmann Models: An Introduction (SpringerVerlag, Berlin, 2000), 308 p.

[10] P.L. Bhatnagar, E.P. Gross and M. Krook, Phys. Rev. 94, 511 (1954).
[11] S. Wolfram, Journal of Statistical Physics 45, 471 (1986).

[12] X. Shan and H. Chen, Physical Review E 47, 1815 (1993).

[13] X. Shan and H. Chen, Physical Review E 49, 2941 (1994).

[14] U. Frisch, B. Hasslacher and Y. Pomeau, Physical Review Letters 56, 1505 (1986).

[15] X. He and L.S. Luo, Physical Review E 56, 6811 (1997).

[16] S. Chapman and T.G. Cowling, The Mathematical Theory of Non-Uniform Gases (Cambridge University Press, Cambridge, 1970), 3rd ed.

[17] N.S. Martys and H. Chen, Physical Review E 53, 743 (1996).

[18] P. d'Humières, D. Lallemand and U. Frish, Europhysics Letters 2, 291 (1986).

[19] R. Shaw, The Dripping Faucet as a Model Chaotic System (Aerial Press, Santa Cruz, 1984). 